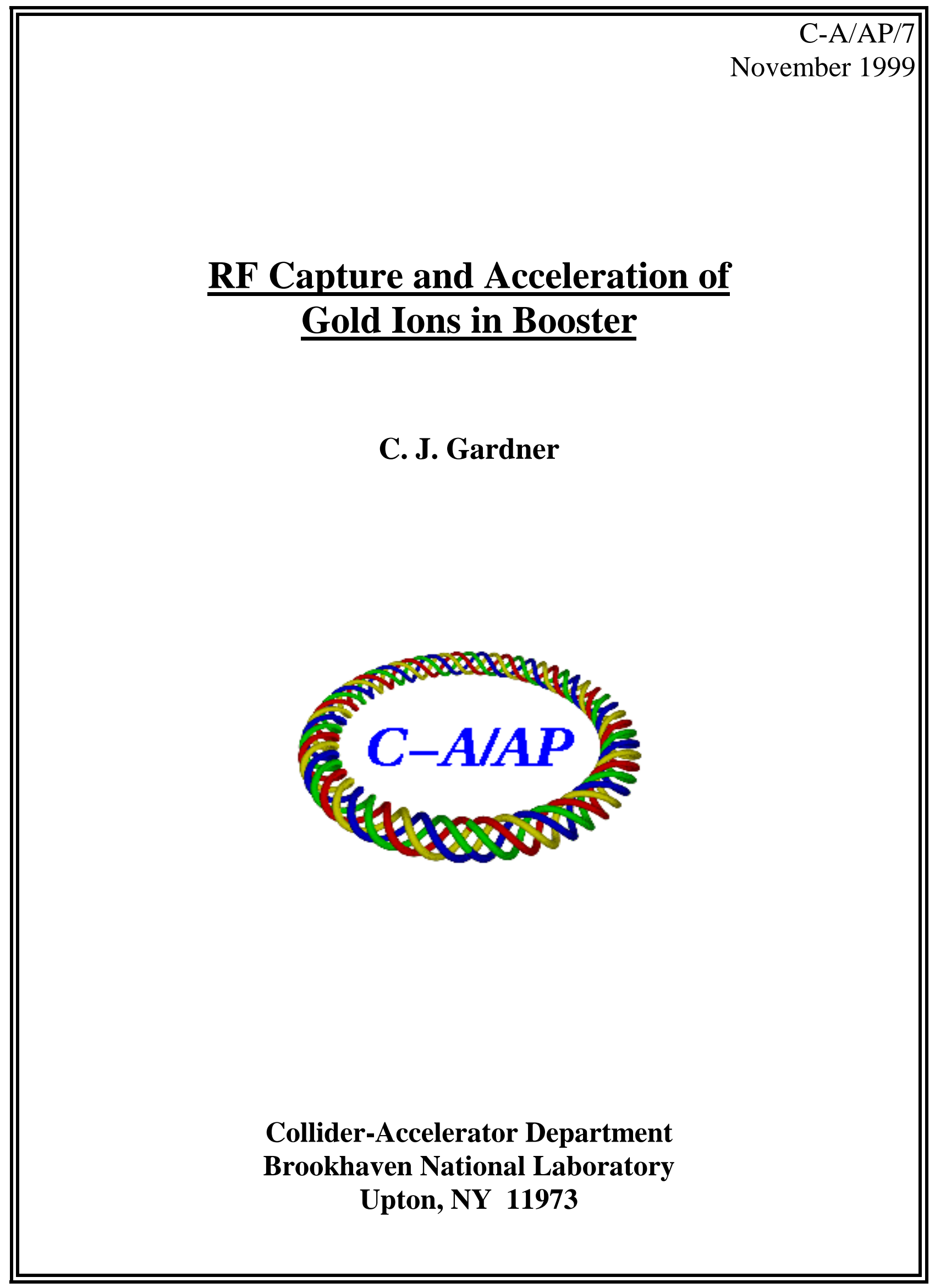




\title{
RF Capture and Acceleration of Gold Ions in Booster
}

\author{
C.J. Gardner
}

November 14, 1999

\section{Introduction}

Following are some notes on the RF capture and acceleration of gold ions $\left(\mathrm{Au}^{31+}\right)$ in the AGS Booster. The basic parameters [1, 2, 3, 4] are reviewed in Sections 2-4. Turn-by-turn simulations of the longitudinal motion for the present setup in Booster are presented in Sections 5-6.

\section{Synchronous Particle and Bucket Parameters}

Let $\rho$ and $R$ be the radius-of-curvature and radius of the design orbit in the Booster, and let $B$ and $R_{s}$ be the magnetic field and radius for the synchronous particle. We assume that $B$ and $R_{s}$ are given throughout the acceleration cycle and calculate the other synchronous particle parameters in terms of these. Thus, the synchronous radius-of-curvature is

$$
\rho_{s}=\rho\left(R_{s} / R\right)^{1 / \alpha}, \quad \alpha=\frac{1}{\gamma_{t}^{2}}
$$

where

$$
\rho=13.8656, \quad R=128.454 / 4
$$

(meters), $\alpha$ is the "momentum compaction" factor and $\gamma_{t}=4.832$ is the transition gamma in the Booster. The synchronous momentum and energy are then

$$
c p_{s}=e Q B \rho_{s}, \quad E_{s}=\sqrt{\left(c p_{s}\right)^{2}+m^{2} c^{4}},
$$

where $e$ is the proton charge and $e Q$ and $m$ are the charge and mass of the particle. (For the present setup [5], gold ions with $Q=31$ and 
$m c^{2}=183.457375 \mathrm{GeV}$, are injected into Booster at a nominal magnetic field of 634.5415 Gauss. This gives $c p_{s}=41.50638 \mathrm{MeV}$ per nucleon.) The synchronous $\beta, \gamma$ and revolution frequency are

$$
\beta_{s}=c p_{s} / E_{s}, \quad \gamma_{s}=E_{s} /\left(m c^{2}\right), \quad f_{s}=c \beta_{s} /\left(2 \pi R_{s}\right) .
$$

We also define the phase slip factor

$$
\eta_{s}=\alpha-\frac{1}{\gamma_{s}^{2}}=\frac{1}{\gamma_{t}^{2}}-\frac{1}{\gamma_{s}^{2}}
$$

The energy gained by the synchronous particle in one turn around the ring is

$$
e Q V_{g} \sin \phi_{s}=2 \pi R_{s} \dot{p}_{s}
$$

where $\phi_{s}$ is the synchronous phase and $V_{g}$ is the vector sum of the maximum voltages across the gaps of the $\mathrm{RF}$ cavities in the ring. (Here and throughout, a dot over a parameter denotes the time derivative.) Using the relation

$$
\frac{\dot{p}_{s}}{p_{s}}=\left(\frac{\dot{B}}{B}+\frac{1}{\alpha} \frac{\dot{R}_{s}}{R_{s}}\right)
$$

we can also write

$$
e Q V_{g} \sin \phi_{s}=2 \pi R_{s} p_{s}\left(\frac{\dot{B}}{B}+\frac{1}{\alpha} \frac{\dot{R}_{s}}{R_{s}}\right)=\frac{E_{s} \beta_{s}^{2}}{f_{s}}\left(\frac{\dot{B}}{B}+\frac{1}{\alpha} \frac{\dot{R}_{s}}{R_{s}}\right) .
$$

One can then obtain $\phi_{s}$ if $V_{g}, B, R_{s}, \dot{B}, \dot{R}_{s}$ are given, and various bucket parameters can then be calculated. The Bucket Half-Height, $\Delta E$, is given by

$$
(\Delta E)^{2}=\frac{e Q V_{g} \beta_{s}^{2} E_{s}}{\pi h \eta_{s}}\left\{\pi \sin \phi_{s}-2 \phi_{s} \sin \phi_{s}-2 \cos \phi_{s}\right\}
$$

and the Bucket Width is

$$
\Delta t=\frac{\left|\pi-\phi_{s}-\phi_{e}\right|}{2 \pi h f_{s}} .
$$

Here $h$ is the RF harmonic number; for the present setup in Booster, $h=6$. The phase $\phi_{e}$ satisfies

$$
\cos \phi_{e}-\cos \left(\pi-\phi_{s}\right)=-\left\{\phi_{e}-\left(\pi-\phi_{s}\right)\right\} \sin \phi_{s} .
$$


For the Stationary Bucket we have either $\phi_{s}=0$ (below transition) or $\phi_{s}=\pi$ (above transition) and the bucket half-height and width become

$$
(\Delta E)_{S}=\left\{\frac{2 e Q V_{g} \beta_{s}^{2} E_{s}}{\pi h\left|\eta_{s}\right|}\right\}^{1 / 2}, \quad(\Delta t)_{S}=\frac{2 \pi}{2 \pi h f_{s}}=\frac{1}{h f_{s}} .
$$

The area of a single Stationary Bucket is

$$
A_{S}=8\left\{\frac{(\Delta E)_{S}}{h \omega_{s}}\right\}=8 \frac{R_{s}}{h c}\left\{\frac{2 e Q V_{g} E_{s}}{\pi h\left|\eta_{s}\right|}\right\}^{1 / 2}
$$

where $\omega_{s}=2 \pi f_{s}=c \beta_{s} / R_{s}$. The area of the corresponding moving bucket is

$$
A=\alpha\left(\phi_{s}\right) A_{S}
$$

where $\alpha\left(\phi_{s}\right)$ is the calculated ratio $A / A_{S}$ for a given stable phase $\phi_{s}$. The function $\alpha\left(\phi_{s}\right)$ is tabulated in Reference [6]; an approximate expression is $[4]$

$$
\alpha\left(\phi_{s}\right) \approx \frac{1-\sin \phi_{s}}{1+\sin \phi_{s}}
$$

For small oscillations about the synchonous phase, the Synchrotron Frequency is

$$
F_{s}=\frac{f_{s}}{\beta_{s}}\left\{\frac{-h \eta_{s} e Q V_{g} \cos \phi_{s}}{2 \pi E_{s}}\right\}^{1 / 2} .
$$

\section{Capture Parameters}

The beam entering the Booster from Tandem is not bunched, and is distributed over the entire length of the ring as the pulse from Tandem is injected. Subsequent capture of beam at all longitudinal positions requires stationary RF buckets with the RF voltage raised adiabatically from zero. In the Booster this is accomplished by "counterphasing" the A3 and B3 $\mathrm{RF}$ cavities so that initially the net voltage seen by the beam is zero. By programming the amount of counterphasing, the net voltage can be raised from zero to some value $V_{c}$ at the end of the capture process. Assuming the energy half-width of the beam entering the Booster is $\delta E$, we can calculate the minimum $V_{c}$ required to capture the beam. The criterion given by Weng [2] is that the (single) stationary bucket area be at least equal to the corresponding area $4 \pi(\delta E) /\left(h \omega_{s}\right)$ occupied by the injected beam. Thus

$$
A_{S}=8\left\{\frac{(\Delta E)_{S}}{h \omega_{s}}\right\} \geq \frac{4 \pi(\delta E)}{h \omega_{s}}
$$


and therefore

$$
(\Delta E)_{S}=\left\{\frac{2 e Q V_{g} \beta_{s}^{2} E_{s}}{\pi h\left|\eta_{s}\right|}\right\}^{1 / 2} \geq \frac{\pi}{2}(\delta E)
$$

Hence, the minimum $V_{c}$ is given by

$$
e V_{g} \geq\left\{\frac{\pi^{3} h\left|\eta_{s}\right|}{8 Q \beta_{s}^{2} E_{s}}\right\}(\delta E)^{2}=\left\{\frac{\pi^{3} h\left|\eta_{s}\right| E_{s}}{8 Q \beta_{s}^{2}}\right\}\left(\delta E / E_{s}\right)^{2}=e V_{c}
$$

Using the relation

$$
\frac{d E}{d p}=\frac{c^{2} p}{E}=c \beta
$$

we can express the energy half-width in terms of the momentum half-width $\delta p$. Thus

$$
\left(\delta E / E_{s}\right)=\beta_{s}^{2}\left(\delta p / p_{s}\right)
$$

and (19) becomes

$$
e V_{c}=\left\{\frac{\pi^{3} h\left|\eta_{s}\right| \beta_{s}^{2} E_{s}}{8 Q}\right\}\left(\delta p / p_{s}\right)^{2}
$$

For $\mathrm{Au}^{31+}$ ions at injection with $h=6$ we have $\left\{\pi^{3} h\left|\eta_{s}\right| \beta_{s}^{2} E_{s}\right\} /(8 Q)=261$ $\mathrm{MeV}$. Assuming $\delta p / p_{s}$ is at most 0.001 , we then find that the minimum $V_{c}$ is at most 261 Volts. (So the voltage required to capture the beam is quite small.) Ideally one would want to raise $V_{g}$ to a higher voltage so that the captured beam ends up occupying the central region of a larger stationary bucket.

We note that since $\phi_{s}=0$ for the stationary bucket, it follows from (8) that

$$
\frac{\dot{R}_{s}}{R_{s}}=-\alpha \frac{\dot{B}}{B}
$$

Thus, if $\phi_{s}=0$ and $\dot{B}>0$ during injection and capture, the radius will decrease and the frequency will increase. For the injection of $\mathrm{Au}^{31+}$ ions in the Booster, Bdot is nominally $1 \mathrm{G} / \mathrm{ms}$ and we have $\alpha \dot{B} / B=6.75 \times 10^{-5}$ per ms. Taking $R_{s}=R=128.454 / 4$ meters, we then find that $\dot{R}_{s}=-2.2$ $\mathrm{mm} / \mathrm{ms}$. If injection and capture without acceleration continue for more than a few ms, the shift in radius therefore can be quite appreciable. In practice, the capture time for $\mathrm{Au}^{31+}$ ions in Booster is 1 to $3 \mathrm{~ms}$. 


\section{Acceleration Parameters}

After capture into stationary buckets with $\dot{B}=1 \mathrm{G} / \mathrm{ms}$, the synchronous phase becomes nonzero and the captured beam is accelerated with Bdot increasing from $1 \mathrm{G} / \mathrm{ms}$ to a maximum of $87 \mathrm{G} / \mathrm{ms}$. Let us assume that $\dot{R}_{s}=0$ during this time. Using the first of equations (3) in (8), we then have

$$
V_{g} \sin \phi_{s}=2 \pi R_{s} \rho_{s} \dot{B} / c
$$

and we see that $V_{g} \sin \phi_{s}$ scales with Bdot only. Employing Gaussian units with $R_{s}=(12845.4 / 4) \mathrm{cm}, \rho_{s}=\rho=1386.56 \mathrm{~cm}, c=2.99792458 \times 10^{10}$ $\mathrm{cm} / \mathrm{s}$, and $\dot{B}=1000 \mathrm{G} / \mathrm{s}$ (which is $1 \mathrm{G} / \mathrm{ms}$ ), we obtain $V_{g} \sin \phi_{s}=0.933223$ Statvolts. Multiplying by 299.792458 to convert to Volts, we then have

$$
V_{g} \sin \phi_{s}=279.773 \text { Volts, } \quad \dot{B}=1 \mathrm{G} / \mathrm{ms} .
$$

At the maximum Bdot we have

$$
V_{g} \sin \phi_{s}=24.3 \mathrm{kV}, \quad \dot{B}=87 \mathrm{G} / \mathrm{ms} .
$$

Now, the maximum $V_{g}$ available for the acceleration of heavy ions in Booster is about $34 \mathrm{kV}$. Using this in (26) we find that at the maximum Bdot,

$$
\sin \phi_{s}=24.3 / 34, \quad \phi_{s}=45.6^{\circ} .
$$

After capture, $V_{g}$ is generally programmed to increase in proportion to Bdot until the maximum of $34 \mathrm{keV}$ is reached; it is then held constant at the maximum value. Thus we have (until the maximum value is reached)

$$
V_{g}=V_{c}+K\left(\dot{B}-\dot{B}_{c}\right)
$$

where $V_{c}$ is value of $V_{g}$ at the end of the capture process and $\dot{B}_{c}$ is the value of $\dot{B}$ during capture. Let us assume that the proportionality constant $K$ is made large enough so that the bucket area increases with time until the maximum $V_{g}$ is reached. At this point the bucket area reaches its maximum value and then decreases as Bdot continues to increase with $V_{g}$ held constant. When Bdot reaches the maximum value of $87 \mathrm{G} / \mathrm{ms}$, the bucket area reaches a minimum value $A^{*}$. This is the so-called bucket area "bottleneck" in the acceleration cycle. For the current setup in Booster, the field at this point is $B^{*}=1.14 \mathrm{kG}$ and the corresponding values of $E_{s}$ and $\eta_{s}$ can be obtained from equations (1-5) 
with $R_{s}=128.454 / 4 \mathrm{~m}$. Using these numbers in (13) and taking $V_{g}=34$ $\mathrm{keV}$, we then obtain the (single) stationary bucket area

$$
A_{S}^{*}=8 \frac{R_{s}}{h c}\left\{\frac{2 e Q V_{g} E_{s}}{\pi h\left|\eta_{s}\right|}\right\}^{1 / 2}=21.0(\mathrm{eV} \mathrm{s})
$$

The stable phase is $45.6^{\circ}$ at this point, so the actual (single) bucket area is

$$
A^{*}=\alpha\left(45.6^{\circ}\right) A_{S}^{*}=(0.157) A_{S}^{*}=3.30(\mathrm{eV} \mathrm{s}) .
$$

This gives an upper limit on the phase-space area that can be captured and accelerated without beam loss. Setting the total phase-space area occupied by the beam at injection equal to $h A^{*}$, we have

$$
\frac{4 \pi}{\omega_{s}} \delta E=\frac{4 \pi}{\omega_{s}} E_{s} \beta_{s}^{2}\left(\delta p / p_{s}\right)=h A^{*}
$$

where $\delta E$ is the energy half-width of the beam and $\omega_{s}, E_{s}, \beta_{s}, p_{s}$ are evaluated at injection. Solving for $\left(\delta p / p_{s}\right)$ we have

$$
\left(\delta p / p_{s}\right)=\frac{h \omega_{s} A^{*}}{4 \pi E_{s} \beta_{s}^{2}}=0.0018
$$

Thus, if $\delta p / p_{s}$ at injection is any larger than 0.0018 , the phase-space area occupied by the beam will be larger than $A^{*}$, and beam will be lost during acceleration.

\section{Turn-by-Turn Equations for Longitudinal Motion}

To calculate the evolution of a given particle distribution during capture and acceleration we need a suitable set of turn-by-turn equations for the longitudinal motion. These are derived here following the treatment of MacLachlan [7]. We consider a ring with a single RF gap and define

$$
T_{n+1}^{s}=T_{n}^{s}+2 \pi / \omega_{n}^{s}, \quad T_{n+1}=T_{n}+2 \pi / \omega_{n} .
$$

Here $T_{n}^{s}$ and $T_{n}$ are respectively the times at which the synchronous and non-synchronous particles make their $n$th pass through the gap; $\omega_{n}^{s}$ and $\omega_{n}$ are the corresponding angular frequencies. Defining

$$
t_{n}=T_{n}-T_{n}^{s}, \quad t_{n+1}=T_{n+1}-T_{n+1}^{s}
$$


we then have

$$
t_{n+1}=t_{n}+2 \pi\left(\frac{1}{\omega_{n}}-\frac{1}{\omega_{n}^{s}}\right)=t_{n}+\frac{2 \pi}{\omega_{n}^{s}}\left(\frac{\omega_{n}^{s}-\omega_{n}}{\omega_{n}}\right) .
$$

Let $\rho$ and $R$ be the radius of curvature and radius of the design orbit, and let $b_{n}^{s}$ and $R_{n}^{s}$ be the field and radius for the synchronous particle on its $n$th turn around the machine. We assume that $b_{n}^{s}$ and $R_{n}^{s}$ are given and then calculate the other parameters of the synchronous particle in terms of these. Thus, the radius-of-curvature, momentum, energy, velocity, angular frequency, and revolution frequency of the synchronous particle just after the $n$th pass through the RF gap are given by

$$
\rho_{n}^{s}=\rho\left(R_{n}^{s} / R\right)^{1 / \alpha}, \quad c p_{n}^{s}=e Q b_{n}^{s} \rho_{n}^{s}, \quad E_{n}^{s}=\sqrt{\left(c p_{n}^{s}\right)^{2}+m^{2} c^{4}}
$$

and

$$
v_{n}^{s}=c \beta_{n}^{s}=c^{2} p_{n}^{s} / E_{n}^{s}, \quad \omega_{n}^{s}=v_{n}^{s} / R_{n}^{s}, \quad 2 \pi f_{n}^{s}=\omega_{n}^{s} .
$$

We also have

$$
\gamma_{n}^{s}=1 / \sqrt{1-\left(\beta_{n}^{s}\right)^{2}}=E_{n}^{s} /\left(m c^{2}\right), \quad \eta_{n}^{s}=\frac{1}{\gamma_{t}^{2}}-\frac{1}{\left(\gamma_{n}^{s}\right)^{2}}
$$

and the synchronous phase is given by

$$
\sin \phi_{n+1}^{s}=\left(E_{n+1}^{s}-E_{n}^{s}\right) /\left(e Q V_{g}\right) .
$$

Similarly, for the non-synchronous particle with energy $E_{n}$, we calculate the momentum, velocity, radius-of-curvature, radius, and angular frequency in terms of $E_{n}$. Thus

$$
c p_{n}=\sqrt{E_{n}^{2}-m^{2} c^{4}}, \quad v_{n}=c^{2} p_{n} / E_{n}
$$

and

$$
\rho_{n}=\frac{c p_{n}}{e Q b_{n}}, \quad R_{n}=R\left(\rho_{n} / \rho\right)^{\alpha}, \quad \omega_{n}=v_{n} / R_{n} .
$$

Here we assume that $b_{n}=b_{n}^{s}$. The turn-by-turn equations for the longitudinal motion of the non-synchronous particle are then

$$
t_{n+1}=t_{n}+2 \pi\left(\frac{1}{\omega_{n}}-\frac{1}{\omega_{n}^{s}}\right)=t_{n}+\frac{2 \pi}{\omega_{n}^{s}}\left(\frac{\omega_{n}^{s}-\omega_{n}}{\omega_{n}}\right)
$$

and

$$
e_{n+1}=e_{n}+e Q V_{g}\left\{\sin \left(\phi_{n+1}+\phi_{n+1}^{s}\right)-\sin \left(\phi_{n+1}^{s}\right)\right\}
$$


where

$$
e_{n}=E_{n}-E_{n}^{s}, \quad e_{n+1}=E_{n+1}-E_{n+1}^{s}
$$

and

$$
\phi_{n+1}=t_{n+1} h \omega_{n+1}^{s} .
$$

Note that the transformation from $\left(t_{n}, e_{n}\right)$ to $\left(t_{n+1}, e_{n+1}\right)$ is symplectic, so the area in $(t, e)$ space is preserved. Let us now define

$$
\phi=h \omega_{n}^{s} t, \quad W=e / \omega_{n}^{s},
$$

where $t$ and $e$ are deviations from the synchronous time and energy. Then the "instantaneous" RF buckets for the $n$th turn are defined by the function $W(\phi)$, where

$$
\begin{gathered}
W^{2}(\phi)=\frac{e Q V_{g}}{a_{n}^{s} \pi}\left\{C_{n+1}^{s}-\cos \left(\phi+\phi_{n+1}^{s}\right)-\phi \sin \left(\phi_{n+1}^{s}\right)\right\}, \\
a_{n}^{s}=\frac{h \eta_{n}^{s}\left(\omega_{n}^{s}\right)^{2}}{\left(\beta_{n}^{s}\right)^{2} E_{n}^{s}}, \quad \eta_{n}^{s}=\frac{1}{\gamma_{t}^{2}}-\frac{1}{\left(\gamma_{n}^{s}\right)^{2}}
\end{gathered}
$$

and

$$
C_{n+1}^{s}=\cos \left(\pi-\phi_{n+1}^{s}\right)+\left(\pi-2 \phi_{n+1}^{s}\right) \sin \phi_{n+1}^{s} .
$$

Using (46) we can obtain the RF bucket in terms of $t$ and $e$. The area of a single bucket on the $n$th turn is

$$
A_{n}=\alpha\left(\phi_{n}^{s}\right) A_{n}^{S}
$$

where

$$
A_{n}^{S}=8 \frac{R_{n}^{s}}{h c}\left\{\frac{2 e Q V_{g} E_{n}^{s}}{\pi h\left|\eta_{n}^{s}\right|}\right\}^{1 / 2} .
$$

Particles that fall outside the RF bucket during acceleration will be lost.

\section{Simulation of Capture and Acceleration}

Using the turn-by-turn equations of the previous section, we simulate the capture and acceleration of an initial distribution of gold ions in Booster. Each particle in the distribution is tracked until it either falls out of the $\mathrm{RF}$ bucket or reaches full energy. 


\subsection{Magnetic Field and Gap-Volt Programs}

The simulation requires magnetic field and gap-volt programs for the acceleration cycle. These are chosen to closely match those actually used in Booster. Although Bdot is nonzero in the actual cycle, we shall assume that during capture, $B$ is held constant with $V_{g}$ increasing linearly from zero to $V_{c}$ in time $T_{c}$. (This is a reasonable approximation since Bdot is small $(1 \mathrm{G} / \mathrm{ms})$ at this time.) Thus, for $0 \leq t \leq T_{c}$, we have

$$
B=B_{c}, \quad \dot{B}=0, \quad V_{g}(t)=\left(V_{c} / T_{c}\right) t
$$

where $B_{c}=634.5415$ (Gauss) is the nominal injection field. After capture, and until the maximum Bdot is reached at time $T^{*}$ (i.e. for $T_{c} \leq t \leq T^{*}$ ), we shall assume that

$$
B(t)=B_{c}+a\left(t-T_{c}\right)^{3}, \quad \dot{B}(t)=3 a\left(t-T_{c}\right)^{2}
$$

and

$$
V_{g}(t)=V_{c}+K \dot{B}(t) .
$$

Here $V_{g}$ increases in proportion to Bdot until the maximum of $34 \mathrm{keV}$ is reached; it is then held constant at the maximum value. The proportionality constant $K$ is an adjustable parameter of the simulation. The parameter $a$ is chosen so that $\dot{B}(t)$ reaches the prescribed maximum value $\dot{B}^{*}$ at time $T^{*}$. Thus

$$
a=\frac{\dot{B}^{*}}{3\left(T^{*}-T_{c}\right)^{2}}
$$

and the field at time $T^{*}$ becomes

$$
B^{*}=B\left(T^{*}\right)=B_{c}+\dot{B}^{*}\left(T^{*}-T_{c}\right) / 3 .
$$

Setting

$$
\dot{B}^{*}=87 \mathrm{G} / \mathrm{ms}, \quad T^{*}-T_{c}=17.4 \mathrm{~ms}
$$

gives a magnetic field cycle which closely matches the one actually used in Booster. After the maximum Bdot is reached, $B$ continues to increase at constant Bdot. Thus, for $t \geq T^{*}$,

$$
B(t)=B^{*}+\dot{B}^{*}\left(t-T^{*}\right)
$$

Using these magnetic field and gap-volt programs we can obtain the turn-by-turn values of the synchronous phase $\left(\phi_{n}^{s}\right)$ and other parameters of 


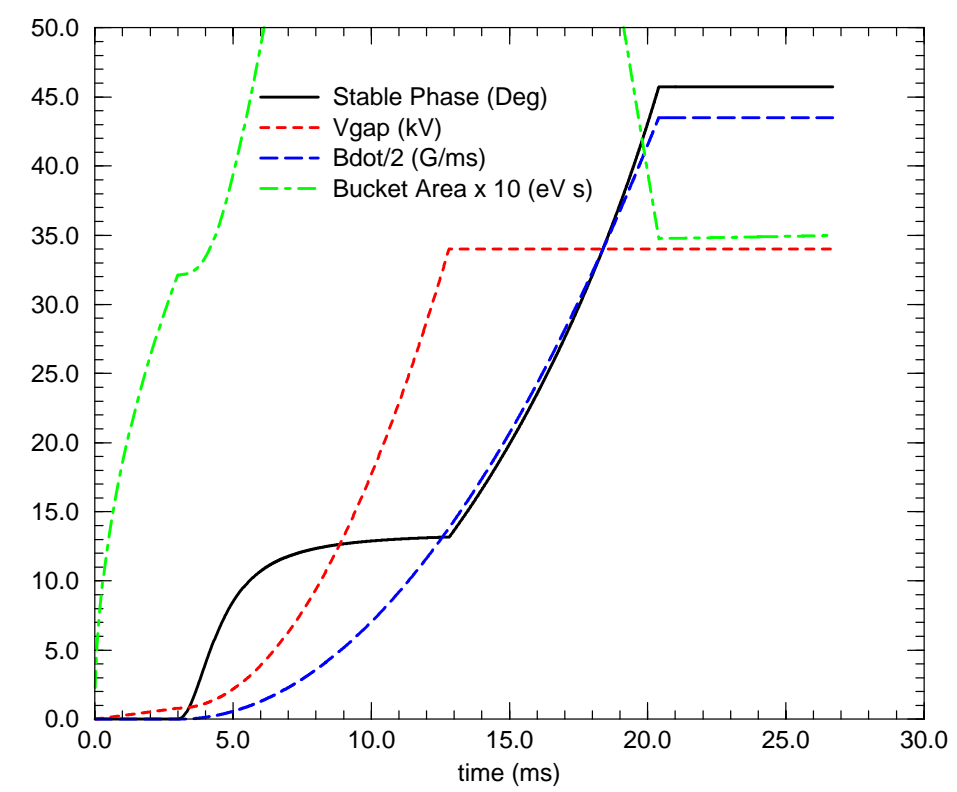

Figure 1: Bdot, Gap Volts, Stable Phase, and Bucket Area with $T_{c}=3 \mathrm{~ms}$, $V_{c}=0.8 \mathrm{kV}$, and $K=1.2 \mathrm{kV} /(\mathrm{G} / \mathrm{ms})$.

the synchronous particle. These can then be used in (50-51) to obtain the bucket area. Fig. 1 shows a plot of $\dot{B}(t), V_{g}(t)$, and the resulting synchronous phase and (single) bucket area for the case in which $T_{c}=3$ $\mathrm{ms}, V_{c}=0.8 \mathrm{kV}$, and $K=1.2 \mathrm{kV} /(\mathrm{G} / \mathrm{ms})$. (Note that what is actually plotted is $\dot{B} / 2$ and 10 times the single bucket area.) Here we see that the bucket area increases (and goes off scale) until $V_{g}(t)$ reaches its maximum value of $34 \mathrm{kV}$; it then decreases to the "bottleneck" at $20.4 \mathrm{~ms}$, after which it slowly increases as $B$ increases with Bdot held constant at 87 $\mathrm{G} / \mathrm{ms}$. If $K$ is too small then the bucket area will decrease immediately after capture as shown in Fig. 2. Here $T_{c}=3 \mathrm{~ms}$ and $V_{c}=0.8 \mathrm{kV}$ as in Fig. 1 , but $K=0.6 \mathrm{kV} /(\mathrm{G} / \mathrm{ms})$. The decrease in bucket area at this point may result in beam loss.

\subsection{Initial Particle Distribution}

The initial particle distribution used in the simulation is an array of points in $(t, e)$ space chosen to cover the distribution of gold beam in Booster just after the beam pulse from tandem has been injected and before RF 


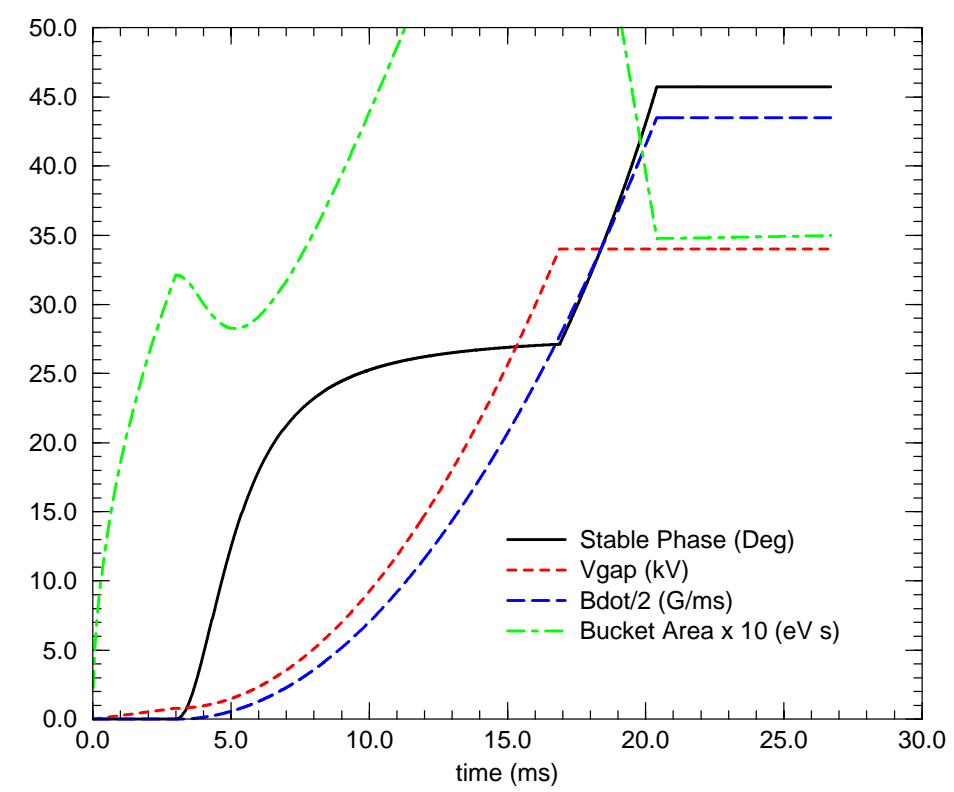

Figure 2: Same as Fig. 1 except $K=0.6 \mathrm{kV} /(\mathrm{G} / \mathrm{ms})$.

capture begins. The beam is unbunched at this time and is spread uniformly around the ring. We shall assume that the distribution of energies within the beam is uniform and is centered on the nominal injection energy. The initial particle distribution is then taken to be a uniform 50-by-50 rectangular array of points in $(t, e)$ space with boundries at $t= \pm \delta t$ and $e= \pm \delta e$. We shall take $\delta e=0.36 \mathrm{MeV}$. This corresponds to the momentum deviation $\delta p$ given by $\delta p / p_{s}=\delta e /\left(E_{s} \beta_{s}^{2}\right)=0.001$ with $p_{s}$, $E_{s}$, and $\beta_{s}$ evaluated at injection. At harmonic $h=6$, the width of a single stationary bucket at injection is $2.518 \mu \mathrm{s}$, so we take $\delta t=1.259 \mu \mathrm{s}$. The resulting array of 2500 points is shown in Fig. 3. The longitudinal emittance of the (single bucket) array is $4(\delta t)(\delta e)=1.813 \mathrm{eV}$-s.

\subsection{Evolution of Particle Distribution}

Tracking each of the 2500 particles of the initial distribution, one obtains the turn-by-turn evolution of the distribution throughout the acceleration cycle. For the magnetic field and gap-volt parameters of Fig. 1, we obtain the distributions and corresponding RF buckets shown in Figures (4-8). (The rectangle of points in the figures is the border of the initial particle 
distribution.) The distribution at the end of Capture is shown in Fig. 4. Here one sees some filamentation of the beam emittance, but all 2500 particles are captured. The distribution at the point of maximum bucket area is shown in Fig. 5. The distribution just after bottleneck is shown in Figures 6 and 7, and the distribution at the full (kinetic) energy of 95.2 $\mathrm{GeV}$ per nucleon is shown in Figure 8. All 2500 particles survive acceleration to full energy.

\subsection{Comments}

Although the momentum spread $\left(\delta p / p_{s}= \pm 0.001\right)$ and (single-bucket) longitudinal emittance $(1.813 \mathrm{eV}-\mathrm{s})$ of the initial particle distribution used in the simulation are (presumably) overestimates of the actual spread and emittance of the Gold beam from Tandem, they serve to define the boundries of the phase-space area that can be accelerated with the present setup in Booster. This is clear from Figures 6 and 7 which show that there is not much room to spare at the bucket area bottleneck.

In Section 4 we showed that, in principle, there is enough bucket area to accommodate the acceleration of an initial particle distribution with $\delta p / p_{s}= \pm 0.0018$ and a (single-bucket) emittance of $3.30 \mathrm{eV}$-s. However, due to emittance filamentation during capture, as shown in Figure 4, a larger bucket is required.

The filamentation that occurs during capture is a critical factor in determining the bucket area requirements for subsequent acceleration not only in Booster but also in AGS and RHIC. The amount of filamentation can be reduced by slowing down the capture process. Figure 9 shows the particle distribution at the end of capture with $T_{c}$ extended to $12 \mathrm{~ms}$. Comparing with Figure 10 (which is the same as Figure 4), we see that there is much less filamentation. Unfortunately, extending the capture time in practice is not compatible with the need to accelerate the beam as

quickly as possible in order to avoid poorly understood loss mechanisms at low energy. 


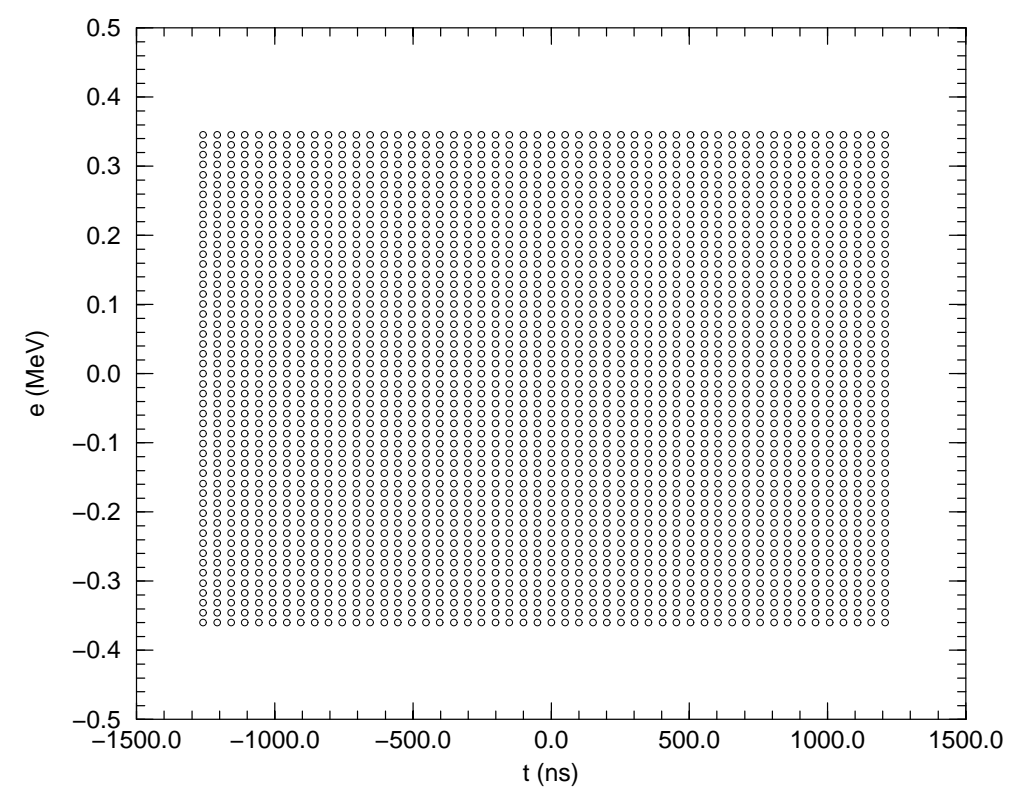

Figure 3: $\mathrm{Au}^{31+}$ Initial Particle Distribution.

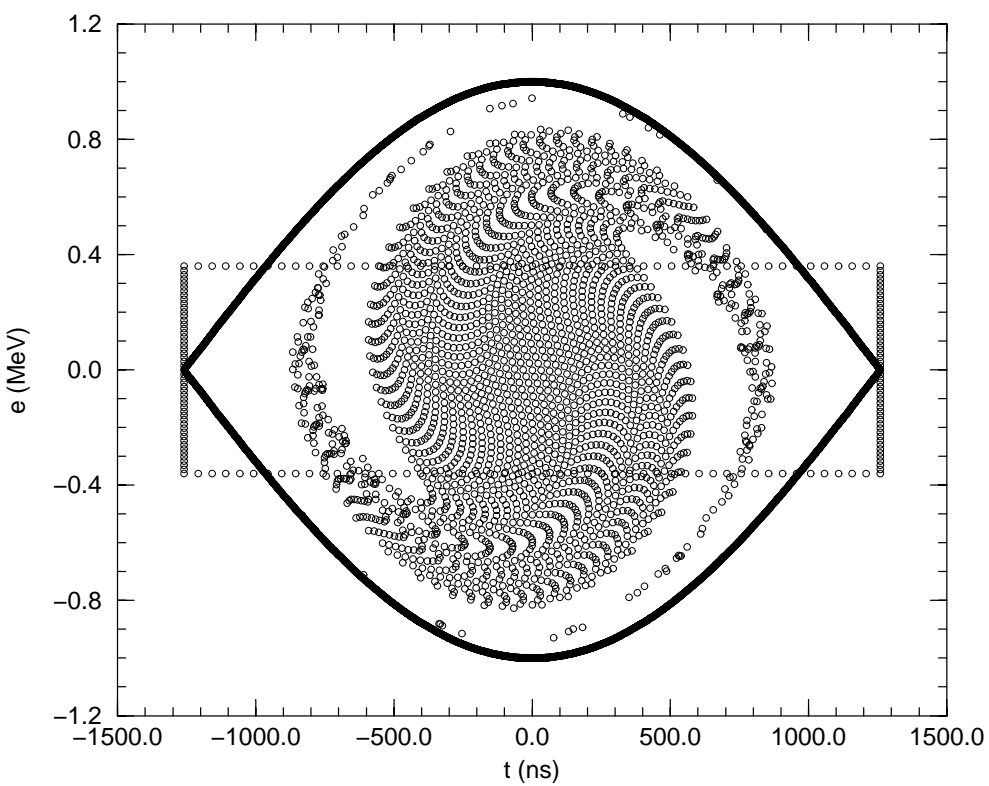

Figure 4: Particle Distribution at end of Capture $\left(T_{c}=3.0 \mathrm{~ms}\right)$. 


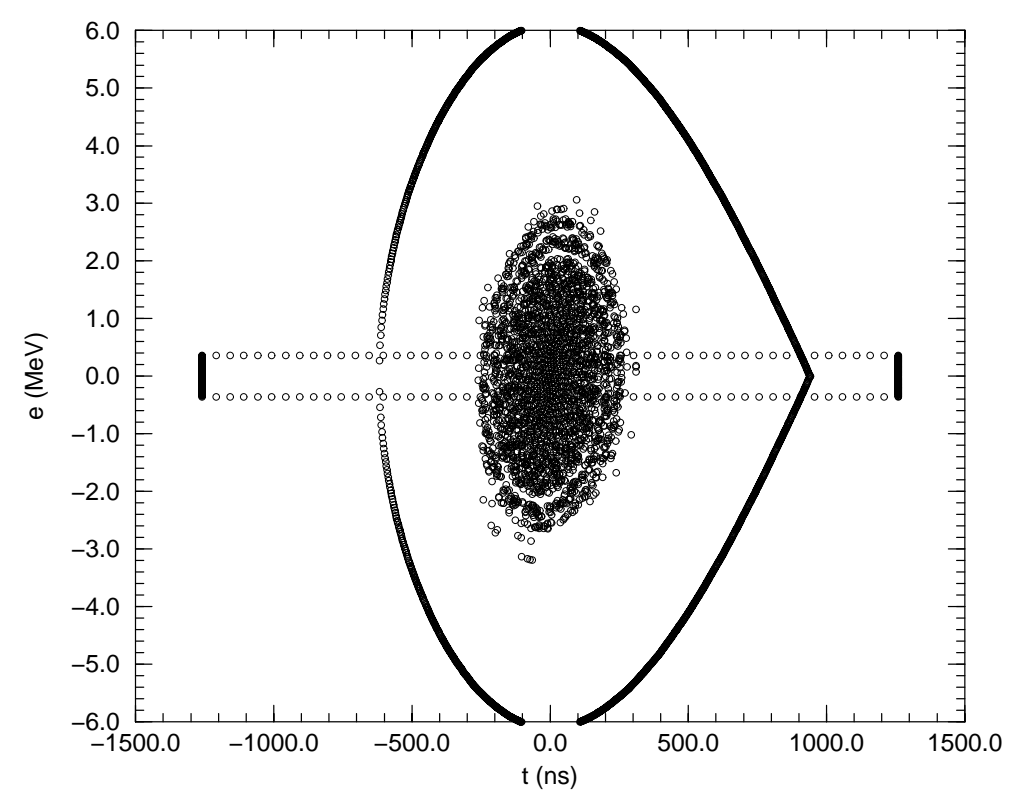

Figure 5: Particle Distribution at point of maximum bucket area.

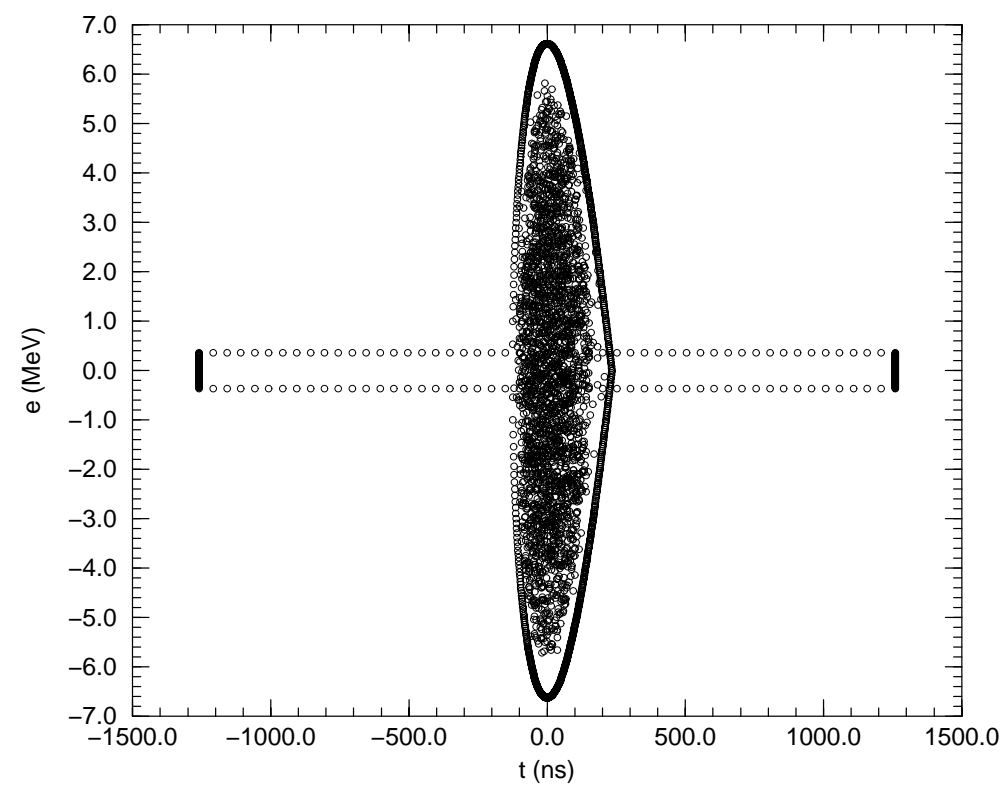

Figure 6: Particle Distribution just after Bottleneck. 


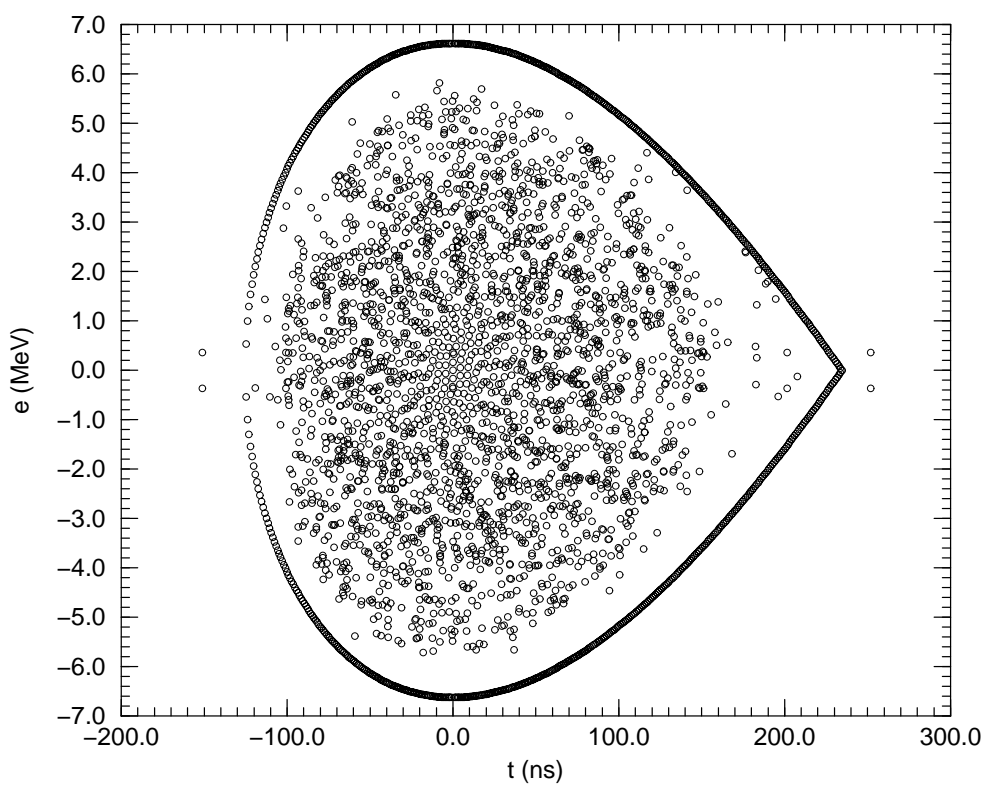

Figure 7: Enlarged View of Fig. 6 (just after Bottleneck).

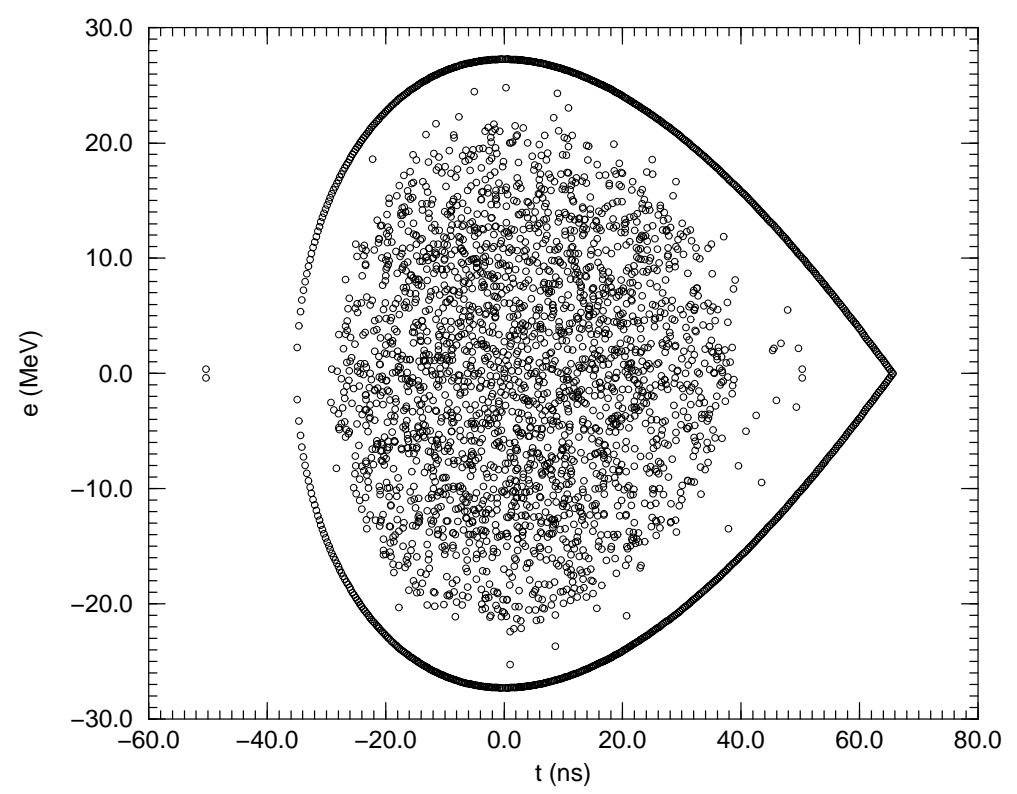

Figure 8: Particle Distribution at Full Energy. 


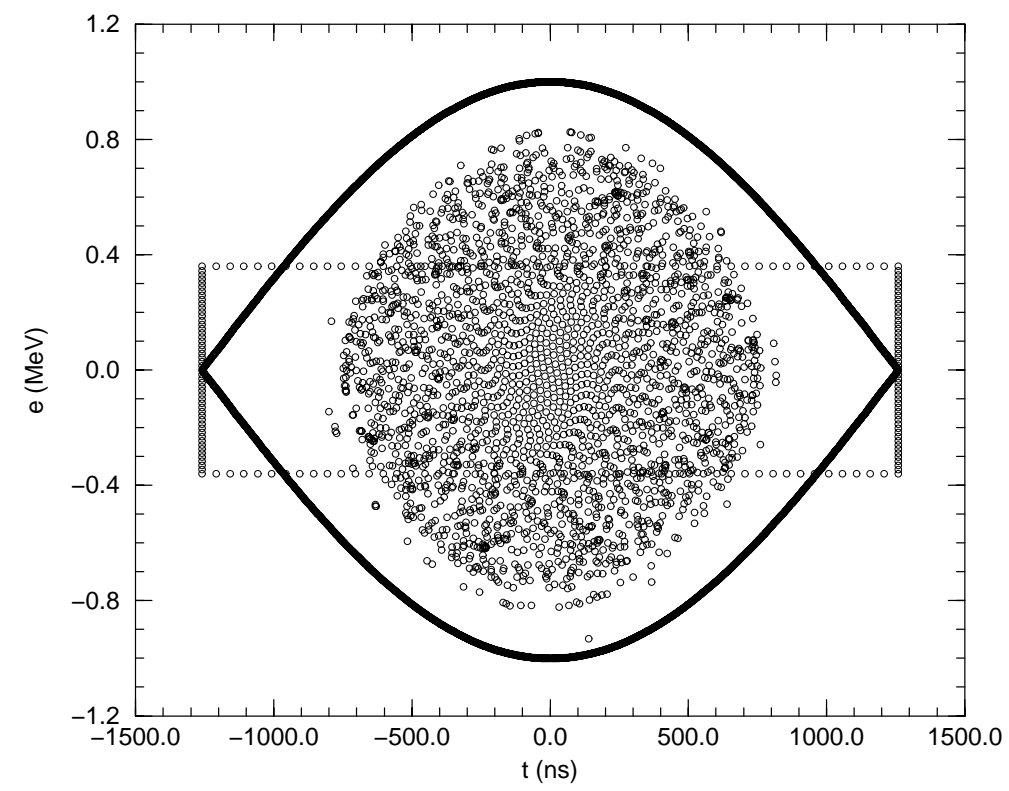

Figure 9: Particle Distribution at end of Capture $\left(T_{c}=12 \mathrm{~ms}\right)$.

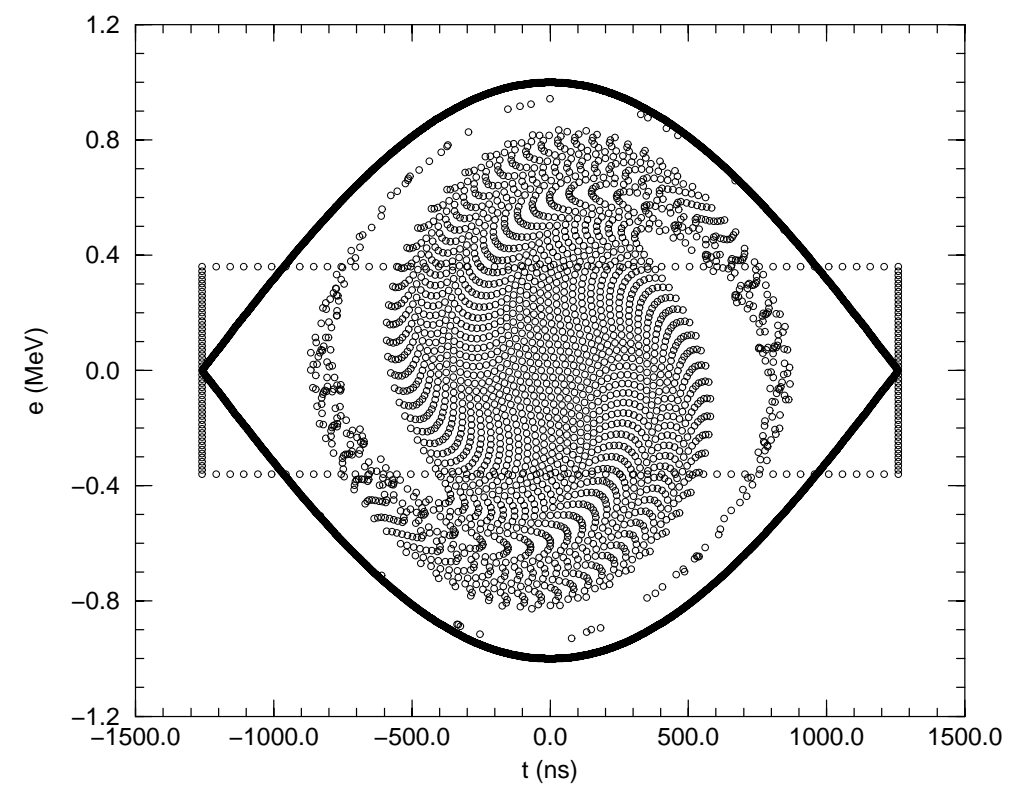

Figure 10: Particle Distribution at end of Capture $\left(T_{c}=3 \mathrm{~ms}\right)$. 


\section{References}

[1] G. Dome, CERN 87-03, 21 April 1987, pp. 110-158

[2] W.T. Weng, Physics of Particle Accelerators, AIP Conference Proceedings 184, New York, 1989, pp.243-287.

[3] M. Conte and W.W. MacKay, "An Introduction to the Physics of Particle Accelerators", World Scientific, 1991.

[4] S.Y. Lee, "Accelerator Physics", World Scientific, 1999.

[5] C.J. Gardner, "Heavy Ion Parameters for 1996", AGS/AD/Tech. Note No. 448, October 10, 1996; "Heavy Ion Parameters for 1997-98, and Some Preliminary Parameters for BAF", AGS/AD/Tech. Note No. 472, November 6, 1997.

[6] C. Bovet, et al., "A Selection of Formulae and Data Useful for the Design of A.G. Synchrotrons", Cern Report CERN/MPS-SI/Int. DL/70/4, 23 April 1970.

[7] J.A. MacLachlan, "Difference Equations for Longitudinal Motion in a Synchrotron", Fermilab internal report FNAL FN-529, December 15, 1989; "Differential Equations for Longitudinal Motion in a Synchrotron", Fermilab internal report FNAL FN-532, January 25, 1990. 